\title{
Analysis of SAR images by multidimensional wavelet transform
}

\author{
J.-P. Ovarlez, L. Vignaud, J.-C. Castelli, M. Tria and M. Benidir
}

\begin{abstract}
The description and development are described of a multidimensional continuous wavelet transform for synthetic aperture radar (SAR) image analysis, especially for the analysis of 'dispersive' reflectors located on a ground illuminated by a SAR. The usual imaging process makes the assumption that the reflectors are isotropic (i.e. that they behave the same way regardless of the angle from which they are viewed) and white (they have the same properties within the emitted frequency bandwidth). New imaging capacities (offering large bandwidth, low band and large angular excursions of analysis, for example) make these assumptions obsolete. The goal is to present methods based on the multidimensional continuous wavelet transform which highlight these effects for target recognition or identification. Some examples of this analysis are given by processing real data collected in X-band by the RAMSES airborne SAR facilities at ONERA.
\end{abstract}

\section{Description of the problem}

Radar imaging allows characterisation of the electromagnetic reflection properties of an illuminated target or ground zone. Information contained in the image is often limited to the spatial distribution of the bright scatterers which reflect a part of the emitted radar signal:

- for instance, radar imaging is used in an anechoic chamber for stealth analysis to identify the parts of the target reflecting the main part of the emitted signal

- in the case of inverse synthetic aperture radar (ISAR) analysis, the goal is to make a spatial image (range and cross-range) of objects (aircraft, satellites) in relative motion with respect to the radar system. This analysis is helpful for recognition and identification purposes

- with airborne or spaceborne SAR, the image of the ground is built from the signal collected by a radar in relative motion with respect to the ground. As for ISAR, these analyses allow recognition and identification tasks to be performed on targets and zones of the ground.

The radar imaging process is often required to deliver information, which is closely connected to the notion of the 'optical' image (infrared image, classical image with CCD system, etc.). Electromagnetic reflection is very different from optical reflection because of the complexity of the electromagnetic wave behaviour in the radar frequency range ( $\mathrm{HF}$ to $\mathrm{W}$-band). Identification or recognition processes using photo-interpretation are currently under

\section{doi: 10.1049/ip-rsn:20030671}

Paper first received 18th December 2002 and in revised form 27th May 2003

J.-P. Ovarlez, L. Vignaud, J.-C. Castelli and M. Tria are with ONERA, DEMR, BP 72, 92322 Chatillon, France. M. Tria is also with L.S.S., Gif-sur-Yvette

M. Benidir is with L.S.S. (UMR 8506), Supelec, 3 rue Joliot Curie, 91192 Gif-sur-Yvette cedex, France development, and it is very difficult to analyse only the spatial images. To improve the identification or recognition process, it is possible to extend the notion of radar imaging by giving other physical information to characterise the object. This confers to the word 'image' a more general meaning.

A first step is to describe the spatial repartition of the object reflectors in a given angular, frequency or polarimetric domain [1]. This work allowed understanding, in the anechoic chamber facilities in ONERA, of the electromagnetic interaction and properties between the different parts of a target (air intake, for example). A second step allows a description also of the kinematical behaviour [2] or the stationarity of reflectors [3] (velocity, acceleration, timevarying reflectors, etc.). The study of polarimetric analysis [4] allows the images to be enriched with information about the electromagnetic behaviour of the object or the soil, and therefore about their characteristics. The expression 'radar image' has to be understood in a wide sense because it can be extended to dimensions other than conventional 2-D metric space.

The study presented here starts from this statement. It takes a part of the description and development of timefrequency methods and continuous wavelet transform for synthetic aperture radar (SAR) image analysis for the study of 'dispersive' scatterers located on terrain illuminated by a SAR. The usual imaging process makes the assumption that the reflectors are isotropic (i.e. that they behave the same way regardless of the angle from which they are viewed) and white (they have the same properties throughout the spectral band emitted). New imaging capacities (offering large bandwidth, low band, large angular excursions of analysis, for example) make the previous assumptions obsolete and it can be brought to the fore in these cases that some degradation of the image quality occurs when performing the standard imaging processes.

This work of characterisation allows us to better understand, evaluate and identify these effects in order to correct or compensate them, and can be of help in the recognition or identification processes. The principles of classical radar imaging are recalled with advantages and drawbacks, 
and wavelet radar imaging is presented. Examples in SAR imaging are obtained from experimental data collected with RAMSES, an airborne SAR facilities in ONERA [5].

\section{Classical radar imaging description}

The backscattering coefficient $H(\overrightarrow{\boldsymbol{k}})$ for a given object illuminated by a radar is characterised, for a distance $R$ going to infinity, as the ratio between the incoming field $E_{r}$ and the emitted field $E_{i}$ (spherical waves)

$$
|H(\overrightarrow{\boldsymbol{k}})|=\lim _{R \rightarrow \infty} \sqrt{4 \pi R^{2}} \frac{E_{r}}{E_{i}}
$$

The square modulus of $H(\overrightarrow{\boldsymbol{k}})$ is called the radar cross-section (RCS) of the object for the wave vector $\overrightarrow{\boldsymbol{k}}$ and is expressed in square metres. The wave vector $\overrightarrow{\boldsymbol{k}}$ is related to the frequency $f$ and to the direction $\theta$ of illumination by relations $|\vec{k}|=2 f / c$ and $\theta=\arg (\overrightarrow{\boldsymbol{k}})$. The model usually used in radar imaging is the model of bright points [6]. The object under analysis can be seen like a set of bright points, i.e. a set of independent sources which reflect in the same way for all frequencies (white points) and all directions of illumination (isotropic points). Let $I(\overrightarrow{\boldsymbol{x}})$ be the amplitude of the bright point located in $\overrightarrow{\boldsymbol{x}}$ in a set of cartesian axes related to the object. The complex backscattering coefficient for the whole object is then given by the in-phase summation of each reflector contribution

$$
H(\overrightarrow{\boldsymbol{k}})=\int I(\overrightarrow{\boldsymbol{x}}) e^{-2 i \pi \overrightarrow{\boldsymbol{k}} \cdot \overrightarrow{\boldsymbol{x}}} d \overrightarrow{\boldsymbol{x}}
$$

Taking the Fourier transform of this coefficient, one can obtain the spatial repartition $I(\overrightarrow{\boldsymbol{x}})$ of the reflectors for a mean frequency (the centre frequency) and for a mean angle of presentation

$$
I(\overrightarrow{\boldsymbol{x}})=\int H(\overrightarrow{\boldsymbol{k}}) e^{2 i \pi \overrightarrow{\boldsymbol{k}} \cdot \overrightarrow{\boldsymbol{x}}} d \overrightarrow{\boldsymbol{k}}
$$

Figure 1 shows the backscattering coefficient $H(\overrightarrow{\boldsymbol{k}})$ of a scaled model aircraft analysed in an anechoic chamber and its spatial repartition of reflectors.

\section{Extended radar imaging}

If the object is illuminated using a broadband signal and/or has a large angular extent, it is obvious that this method, with the strong assumption of white and isotropic bright points, is no longer sufficient. Removing this assumption, the repartition density of reflectors $I(\overrightarrow{\boldsymbol{x}})$ must depend on the wave vector $\overrightarrow{\boldsymbol{k}}$ and must now be noted $I(\overrightarrow{\boldsymbol{x}}, \overrightarrow{\boldsymbol{k}})$. This makes impossible the inversion of the relation (2) by the classical Fourier transform.

Time-frequency analysis and the physical group theory allow the construction of extended radar images $[1,7,8]$. The dimension of these images, called hyperimages [9], is the product of the dimension of the vector $\overrightarrow{\boldsymbol{x}}$ by the dimension of the vector $\overrightarrow{\boldsymbol{k}}$. Figure 2 shows a bidimensional example of this kind of extended analysis of the backscattering coefficient presented in Fig. 1. Then, the frequency behaviour and the angular illumination of each reflector located in $\overrightarrow{\boldsymbol{x}}$ can be obtained.

The principle of the extended radar imaging [1] is based on a physical group of transformations, the similarity group $S$. This is acting on physical variables $\overrightarrow{\boldsymbol{x}}$ and $\overrightarrow{\boldsymbol{k}}$ by rotations $\mathcal{R}_{\theta}$, dilations $a$ in length (or time) and translations $\delta \overrightarrow{\boldsymbol{x}}$ according to

$$
\begin{aligned}
& \overrightarrow{\boldsymbol{x}} \rightarrow \overrightarrow{\boldsymbol{x}}^{\prime}=a \mathcal{R}_{\theta} \overrightarrow{\boldsymbol{x}}+\delta \overrightarrow{\boldsymbol{x}} \\
& \downarrow \\
& \overrightarrow{\boldsymbol{k}} \rightarrow \overrightarrow{\boldsymbol{k}}^{\prime}=a^{-1} \mathcal{R}_{\theta} \overrightarrow{\boldsymbol{k}}
\end{aligned}
$$

The transformation law of the backscattering coefficient $H(\overrightarrow{\boldsymbol{k}})$ and its extended image $I(\overrightarrow{\boldsymbol{x}}, \overrightarrow{\boldsymbol{k}})$ in a change of such a reference system is therefore given by

$$
\begin{gathered}
H(\overrightarrow{\boldsymbol{k}}) \rightarrow H^{\prime}(\overrightarrow{\boldsymbol{k}})=a e^{-2 i \pi \overrightarrow{\boldsymbol{k}} \cdot \delta \overrightarrow{\boldsymbol{x}}} H\left(a \mathcal{R}_{\theta}^{-1} \overrightarrow{\boldsymbol{k}}\right) \\
\downarrow \\
\downarrow \\
\begin{array}{l}
\downarrow \\
(\overrightarrow{\boldsymbol{x}}, \overrightarrow{\boldsymbol{k}})
\end{array}
\end{gathered}
$$

\subsection{General construction of the extended images}

To construct radar images, a first approach consists in representing the image as a Hermitian and bilinear form of the backscattering coefficient

$$
I(\overrightarrow{\boldsymbol{x}}, \overrightarrow{\boldsymbol{k}})=\iint K\left(\overrightarrow{\boldsymbol{k}}_{1}, \overrightarrow{\boldsymbol{k}}_{2} ; \overrightarrow{\boldsymbol{x}}, \overrightarrow{\boldsymbol{k}}\right) H\left(\overrightarrow{\boldsymbol{k}}_{1}\right) H^{*}\left(\overrightarrow{\boldsymbol{k}}_{2}\right) d \overrightarrow{\boldsymbol{k}}_{1} d \overrightarrow{\boldsymbol{k}}_{2}
$$

where $I(\overrightarrow{\boldsymbol{x}}, \overrightarrow{\boldsymbol{k}})$ represents a spectral image of the target and where the kernel $K\left(\overrightarrow{\boldsymbol{k}}_{1}, \overrightarrow{\boldsymbol{k}}_{2} ; \overrightarrow{\boldsymbol{x}}, \overrightarrow{\boldsymbol{k}}\right)$ is supposed to be Hermitian. This kernel is not known but can be determined with some physical constraints made on the image

- The image can satisfy the property of covariance by the similarity group $S$ given by (5)
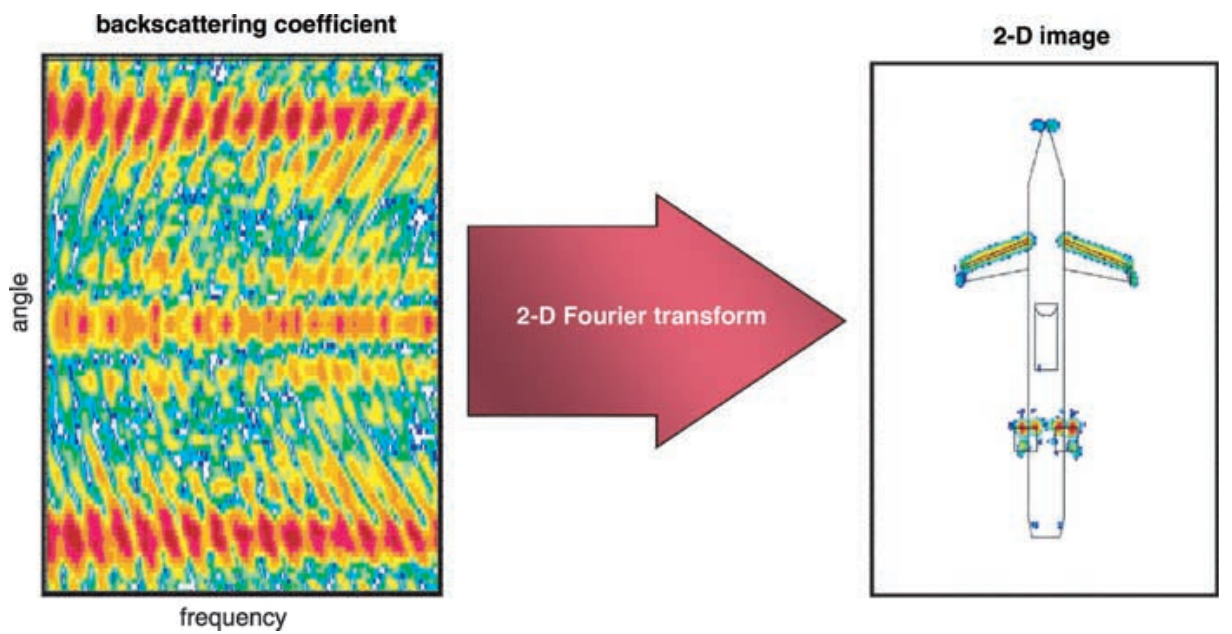

Fig. 1 Classical bidimensional radar imaging of aircraft scaled model 


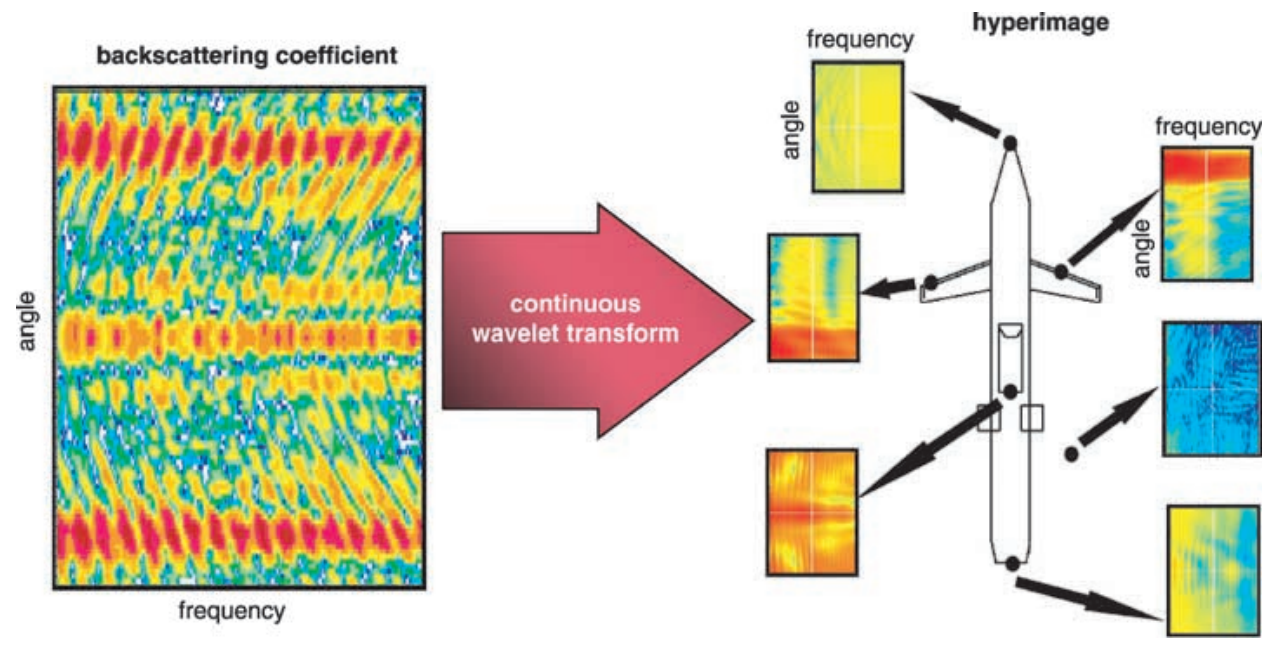

Fig. 2 Extended bidimensional radar imaging of aircraft scaled model

- The image of the spatial bright points distribution in $\mathrm{IR}^{2}$ can be seen as a positive density, such as its integral on some surface $\mathcal{D}$ represents the RCS (radar cross-section) contribution $\sigma_{\mathcal{D}}(\overrightarrow{\boldsymbol{k}})$ of all the reflectors contained in $\mathcal{D}$

$$
\sigma_{\mathcal{D}}(\overrightarrow{\boldsymbol{k}})=\int_{\mathcal{D}} I(\overrightarrow{\boldsymbol{x}}, \overrightarrow{\boldsymbol{k}}) d \overrightarrow{\boldsymbol{x}}
$$

- If $\mathcal{D}$ represents the whole plan, the image can respect the well known marginal property

$$
\int I(\overrightarrow{\boldsymbol{x}}, \overrightarrow{\boldsymbol{k}}) d \overrightarrow{\boldsymbol{x}}=|H(\overrightarrow{\boldsymbol{k}})|^{2}
$$

- The energy conservation between the image space and backscattering coefficient space leads to an important relation (Moyal formula) which connects the inner product between two given backscattering coefficients $H_{1}$ and $H_{2}$ and their associated images $I_{1}$ and $I_{2}$

$$
\left|\int H_{1}(\overrightarrow{\boldsymbol{k}}) H_{2}^{*}(\overrightarrow{\boldsymbol{k}}) d \overrightarrow{\boldsymbol{k}}\right|^{2}=\iint I_{1}(\overrightarrow{\boldsymbol{x}}, \overrightarrow{\boldsymbol{k}}) I_{2}^{*}(\overrightarrow{\boldsymbol{x}}, \overrightarrow{\boldsymbol{k}}) d \overrightarrow{\boldsymbol{x}} d \overrightarrow{\boldsymbol{k}}
$$

The time-frequency analysis has shown that no distribution can satisfy all these properties. For example, the property (9) does not always allow us to obtain an image everywhere positive, which is inconsistent with the RCS nature of the image given by (7) or (8).

The second approach is thus to construct a regularised form of these images which overcomes this drawback but acts to the detriment of the marginalisation property. This second approach is developed in the next Section.

\subsection{Construction of the extended images by wavelet transform}

One can view the construction of the extended images by two similar methods. The first consists in smoothing the general distribution given by (6). The second allows interpretation of the extended images by a mean of localised states.

\subsubsection{Construction by smoothed bilinear} distribution: Let $\phi(\overrightarrow{\boldsymbol{k}})$ be a backscattering function for a reference object. This distribution is supposed to be well located around the origin $\overrightarrow{\boldsymbol{x}}=\overrightarrow{\mathbf{0}}$, to reflect mainly in the direction of the unit vector $\overrightarrow{\boldsymbol{n}}=\overrightarrow{\boldsymbol{k}} /|\overrightarrow{\boldsymbol{k}}|$ for the frequency $k=1$ and to be invariant by rotation around the $\overrightarrow{\boldsymbol{n}}$ axis. By the action of the group $S$, a family of wavelet bases
$\Psi_{\overrightarrow{\boldsymbol{x}}_{0}, \overrightarrow{\boldsymbol{k}}_{0}}(\overrightarrow{\boldsymbol{k}})$ can be generated from the mother wavelet $\phi(k, \theta)$ according to

$$
\begin{aligned}
\Psi_{\overrightarrow{\boldsymbol{x}}_{0}, \overrightarrow{\boldsymbol{k}}_{0}}(\overrightarrow{\boldsymbol{k}}) & =\frac{1}{k_{0}} e^{-2 i \pi \overrightarrow{\boldsymbol{k}} \cdot \overrightarrow{\boldsymbol{x}}_{0}} \phi\left(\frac{1}{k_{0}} \mathcal{R}_{\theta_{0}}^{-1} \overrightarrow{\boldsymbol{k}}\right) \\
& =\frac{1}{k_{0}} e^{-2 i \pi \overrightarrow{\boldsymbol{k}} \cdot \overrightarrow{\boldsymbol{x}}_{0}} \phi\left(\frac{k}{k_{0}}, \theta-\theta_{0}\right)
\end{aligned}
$$

An image $\tilde{\boldsymbol{I}}\left(\overrightarrow{\boldsymbol{x}}_{0}, \overrightarrow{\boldsymbol{k}}_{0}\right)$ can be constructed using the Moyal formula (9), the covariance property (5) with $H_{1}(\overrightarrow{\boldsymbol{k}})=$ $H(\overrightarrow{\boldsymbol{k}}), H_{2}(\overrightarrow{\boldsymbol{k}})=\Psi_{\overrightarrow{\boldsymbol{x}}_{0}, \overrightarrow{\boldsymbol{k}}_{0}}(\overrightarrow{\boldsymbol{k}}), I_{1}=I_{H}$ and $I_{2}=I_{\phi}$. This leads to

$$
\begin{aligned}
\tilde{I}\left(\overrightarrow{\boldsymbol{x}}_{0}, \overrightarrow{\boldsymbol{k}}_{0}\right) & =\iint I_{H}(\overrightarrow{\boldsymbol{x}}, \overrightarrow{\boldsymbol{k}}) I_{\phi}^{*}\left(k_{0} \mathcal{R}_{\theta_{0}}^{-1}\left(\overrightarrow{\boldsymbol{x}}-\overrightarrow{\boldsymbol{x}}_{0}\right), \frac{1}{k_{0}} \mathcal{R}_{\theta_{0}}^{-1} \overrightarrow{\boldsymbol{k}}\right) d \overrightarrow{\boldsymbol{x}} d \overrightarrow{\boldsymbol{k}} \\
& =\left|\int H(\overrightarrow{\boldsymbol{k}}) \frac{1}{k_{0}} e^{2 i \pi \overrightarrow{\boldsymbol{k}} \cdot \overrightarrow{\boldsymbol{x}}_{0}} \phi^{*}\left(\frac{1}{k_{0}} \mathcal{R}_{\theta_{0}}^{-1} \overrightarrow{\boldsymbol{k}}\right) d \overrightarrow{\boldsymbol{k}}\right|^{2}
\end{aligned}
$$

The right-hand side is nothing but the wavelet coefficient $C\left(\overrightarrow{\boldsymbol{x}}_{0}, \overrightarrow{\boldsymbol{k}}_{0}\right)$ which is introduced as the invariant scalar product of the group $S$ between the backscattering coefficient $H$ and each element $\Psi_{\overrightarrow{\boldsymbol{x}}_{0}, \overrightarrow{\boldsymbol{k}}_{0}}$ of the wavelet basis

$$
\begin{aligned}
C\left(\overrightarrow{\boldsymbol{x}}_{0}, \overrightarrow{\boldsymbol{k}}_{0}\right) & =\int H(\overrightarrow{\boldsymbol{k}}) \Psi_{\overrightarrow{\boldsymbol{x}}_{0}, \overrightarrow{\boldsymbol{k}}_{0}}^{*}(\overrightarrow{\boldsymbol{k}}) d \overrightarrow{\boldsymbol{k}} \\
& =\int_{0}^{2 \pi} d \theta \int_{0}^{+\infty} k H(k, \theta) \Psi_{\overrightarrow{\boldsymbol{x}}_{0}, \overrightarrow{\boldsymbol{k}}_{0}}^{*}(\overrightarrow{\boldsymbol{k}}) d k
\end{aligned}
$$

The reconstruction property allows us to recover the signal with the knowledge of its wavelet coefficients

$$
H(\overrightarrow{\boldsymbol{k}})=\frac{1}{K(\phi)} \int d \overrightarrow{\boldsymbol{x}}_{0} \int C\left(\overrightarrow{\boldsymbol{x}}_{0}, \overrightarrow{\boldsymbol{k}}_{0}\right) \Psi_{\overrightarrow{\boldsymbol{x}}_{0}, \overrightarrow{\boldsymbol{k}}_{0}}(\overrightarrow{\boldsymbol{k}}) d \overrightarrow{\boldsymbol{k}}_{0}
$$

where $K(\phi)$ is the admissibility coefficient defined as

$$
K(\phi)=\int \frac{|\phi(\overrightarrow{\boldsymbol{k}})|^{2}}{k^{2}} d \overrightarrow{\boldsymbol{k}}<\infty
$$

\subsubsection{Probabilistic mean of localised} states: The isometry property gives a probabilistic sense to the square modulus of the wavelet coefficient

$$
\int|H(\overrightarrow{\boldsymbol{k}})|^{2} d \overrightarrow{\boldsymbol{k}}=\iint \frac{1}{K(\phi)}\left|C\left(\overrightarrow{\boldsymbol{x}}_{0}, \overrightarrow{\boldsymbol{k}}_{0}\right)\right|^{2} d \overrightarrow{\boldsymbol{x}}_{0} d \overrightarrow{\boldsymbol{k}}_{0}
$$




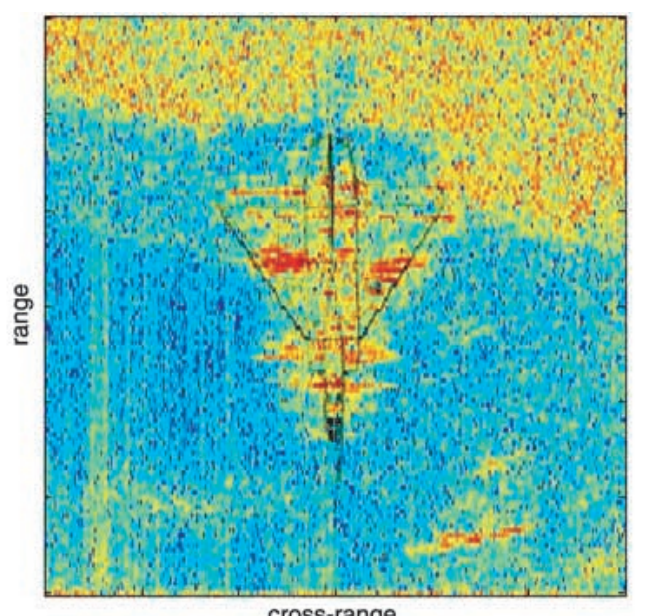

cross-range

a

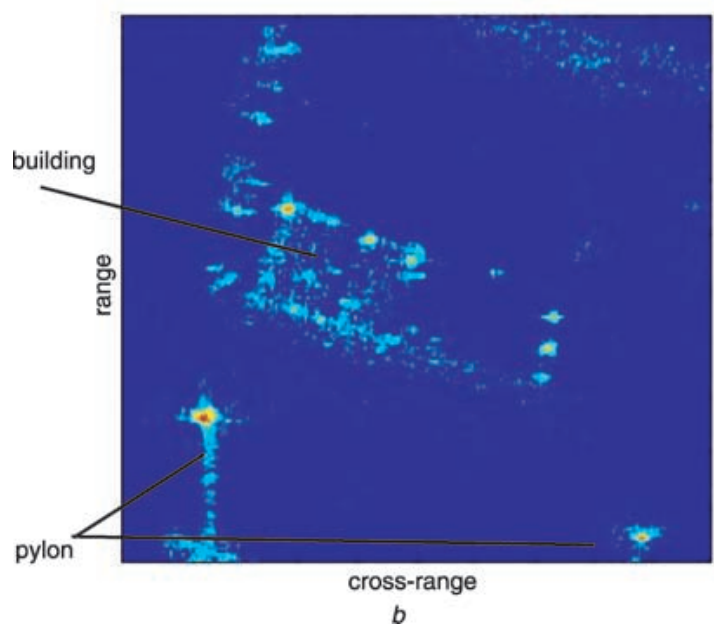

$b$

Fig. 3 SAR images of aircraft type target (a) and building with some pylons around (b) built by the RMA algorithm All the information relative to frequency or angular sensitivity is lost

This coefficient can be seen as a probability density in the whole $(\overrightarrow{\boldsymbol{x}}, \overrightarrow{\boldsymbol{k}})$ space. Among all the images $I(\overrightarrow{\boldsymbol{x}}, \overrightarrow{\boldsymbol{k}})$, the only distribution $L_{\overrightarrow{\boldsymbol{x}}_{0}, \overrightarrow{\boldsymbol{k}}_{0}}$ which is perfectly located in $\left(\overrightarrow{\boldsymbol{x}}_{0}, \overrightarrow{\boldsymbol{k}}_{0}\right)$ and which is still located after the action of the similarity group
$S$ in $\left(a \mathcal{R} \overrightarrow{\boldsymbol{x}}_{0}+\delta \overrightarrow{\boldsymbol{x}}, a^{-1} \mathcal{R} \overrightarrow{\boldsymbol{k}}_{0}\right)$ must verify the following equality:

$$
L_{a \mathcal{R} \overrightarrow{\boldsymbol{x}}_{0}+\delta \overrightarrow{\boldsymbol{x}}, a^{-1} \mathcal{R} \overrightarrow{\boldsymbol{k}}_{0}}(\overrightarrow{\boldsymbol{x}}, \overrightarrow{\boldsymbol{k}})=L_{\overrightarrow{\boldsymbol{x}}_{0}, \overrightarrow{\boldsymbol{k}}_{0}}\left(a^{-1} \mathcal{R}^{-1}(\overrightarrow{\boldsymbol{x}}-\delta \overrightarrow{\boldsymbol{x}}), a \mathcal{R}^{-1} \overrightarrow{\boldsymbol{k}}\right)
$$

Solutions of (17) are given by

$$
L_{\overrightarrow{\boldsymbol{x}}_{0}, \overrightarrow{\boldsymbol{k}}_{0}}(\overrightarrow{\boldsymbol{x}}, \overrightarrow{\boldsymbol{k}})=A \delta\left(\overrightarrow{\boldsymbol{x}}-\overrightarrow{\boldsymbol{x}}_{0}\right) \delta\left(\overrightarrow{\boldsymbol{k}}-\overrightarrow{\boldsymbol{k}}_{0}\right)
$$

where $\delta$ is a Dirac distribution and $A$ a real coefficient.

This second approach consists in considering the radar images as a mean of localised states $L_{\overrightarrow{\boldsymbol{x}}_{0}, \overrightarrow{\boldsymbol{k}}_{0}}(\overrightarrow{\boldsymbol{x}}, \overrightarrow{\boldsymbol{k}})$ given by (18) with the density given by $p\left(\overrightarrow{\boldsymbol{x}}_{0}, \overrightarrow{\boldsymbol{k}}_{0}\right)=\left|C\left(\overrightarrow{\boldsymbol{x}}_{0}, \overrightarrow{\boldsymbol{k}}_{0}\right)\right|^{2} / K(\phi)$. This leads to

$$
I(\overrightarrow{\boldsymbol{x}}, \overrightarrow{\boldsymbol{k}})=\int \frac{1}{K(\phi)}\left|C\left(\overrightarrow{\boldsymbol{x}}_{0}, \overrightarrow{\boldsymbol{k}}_{0}\right)\right|^{2} L_{\overrightarrow{\boldsymbol{x}}_{0}, \boldsymbol{k}_{0}}(\overrightarrow{\boldsymbol{x}}, \overrightarrow{\boldsymbol{k}}) d \overrightarrow{\boldsymbol{k}}_{0} d \overrightarrow{\boldsymbol{x}}_{0}
$$

The radar image is therefore defined as

$$
I(\overrightarrow{\boldsymbol{x}}, \overrightarrow{\boldsymbol{k}})=\frac{1}{K(\phi)}|C(\overrightarrow{\boldsymbol{x}}, \overrightarrow{\boldsymbol{k}})|^{2}
$$

\subsubsection{Interpretation of the wavelet coeffi-} cient: The last two methods give exactly the same interpretation. It follows from (13) and (20) that the kernel $K$ in (6) is given by the specular choice

$$
\begin{aligned}
K\left(\overrightarrow{\boldsymbol{k}}_{1}, \overrightarrow{\boldsymbol{k}}_{2} ; \overrightarrow{\boldsymbol{x}}, \overrightarrow{\boldsymbol{k}}\right)= & \frac{1}{K(\phi)} \frac{k_{1} k_{2}}{k^{2}} e^{2 i \pi\left(\overrightarrow{\boldsymbol{k}}_{1}-\overrightarrow{\boldsymbol{k}}_{2}\right) \overrightarrow{\boldsymbol{x}}^{*}} \phi^{*} \\
& \times\left(\frac{1}{k} \mathcal{R}_{\theta}^{-1} \overrightarrow{\boldsymbol{k}}_{1}\right) \phi\left(\frac{1}{k} \mathcal{R}_{\theta}^{-1} \overrightarrow{\boldsymbol{k}}_{2}\right)
\end{aligned}
$$

For each frequency and each angle of illumination, a spatial repartition of reflectors which respond at this frequency and this angle can be defined. Inversely, for each reflector location, it is possible to analyse its behaviour in the frequency and in the angular domain. Finally, for a given wave vector $\overrightarrow{\boldsymbol{k}}$, the quantity $I(\overrightarrow{\boldsymbol{x}}, \overrightarrow{\boldsymbol{k}}) \Delta \overrightarrow{\boldsymbol{x}}$ represents the RCS level of the reflector located in $\overrightarrow{\boldsymbol{x}}$ for a given vicinity $\Delta \overrightarrow{\boldsymbol{x}}$ of $\overrightarrow{\boldsymbol{x}}$.

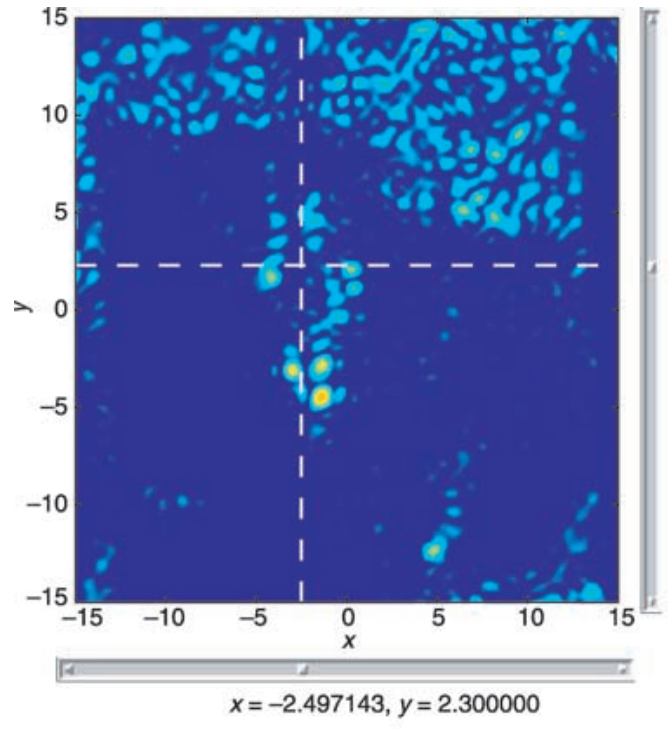

a

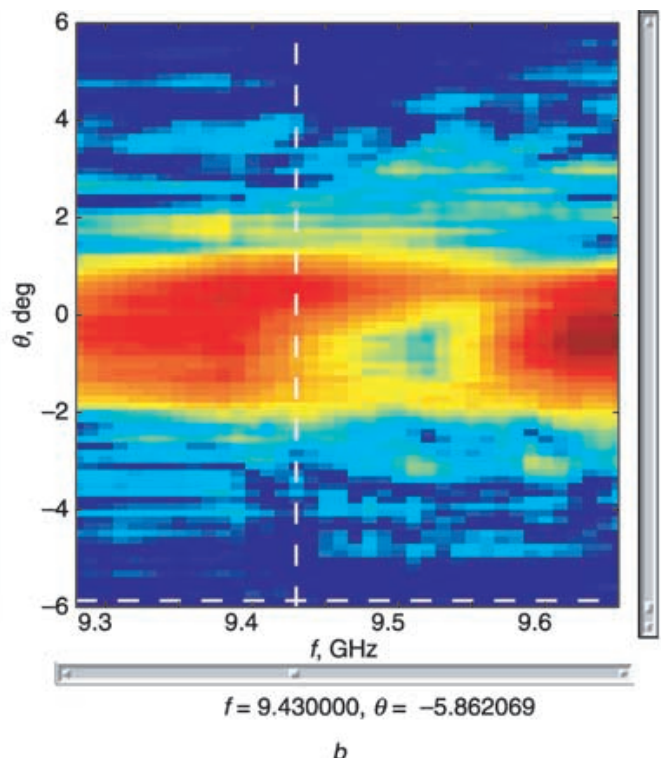

b

Fig. 4 Variation of spatial SAR image for a given angle $\theta=-5.86^{\circ}$ and frequency $f=9.43 \mathrm{GHz}$

a Image $(x, y)$

$b$ Image $(f, \theta)$ 

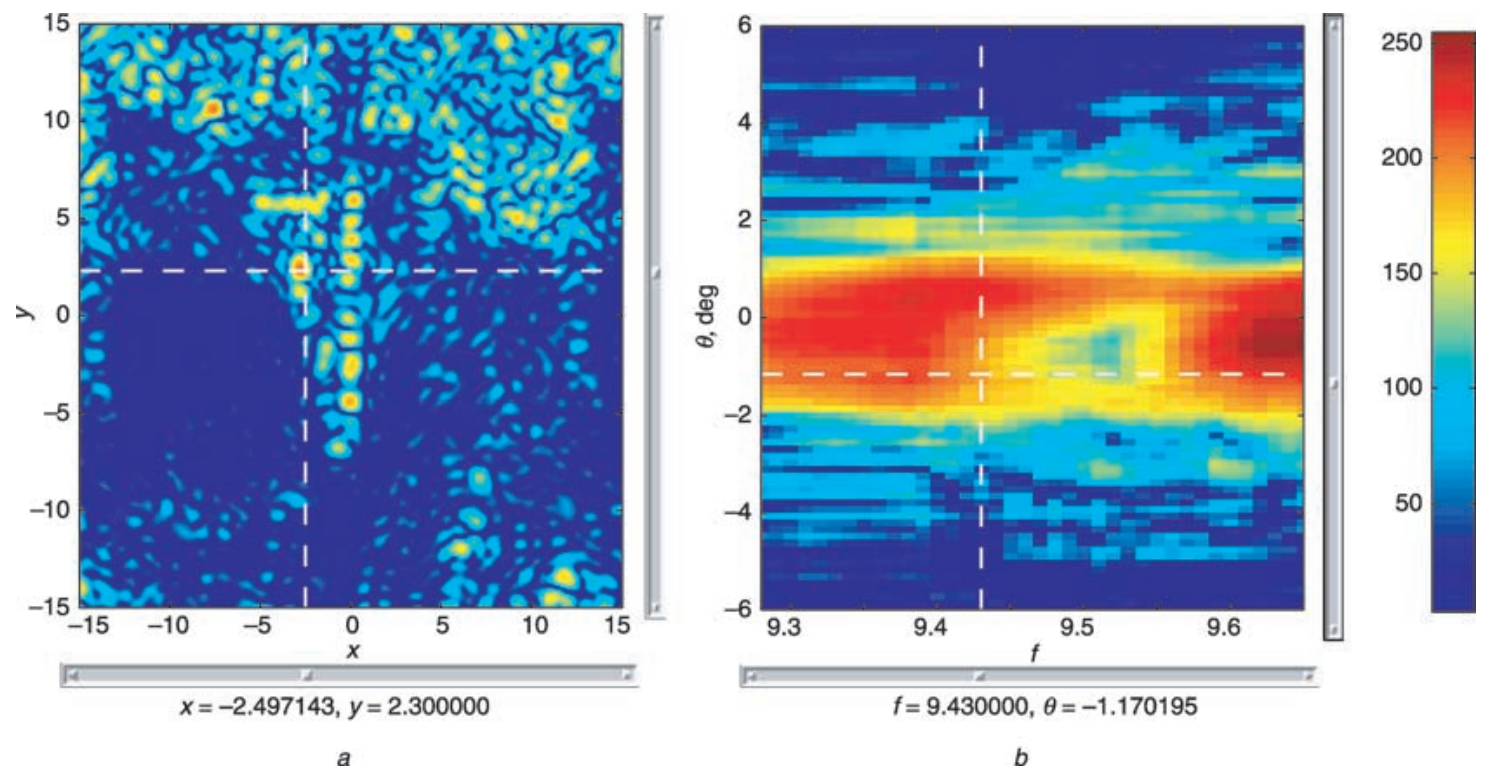

Fig. 5 Variation of spatial SAR image for a given angle $\theta=-1.17^{\circ}$ and frequency $f=9.43 \mathrm{GHz}$

$a$ Image $(x, y)$

$b$ Image $(f, \theta)$

To analyse this 4-D structure, a visual display interface called 'i4D' [10] has been developed and allows an interactive and dynamic analysis to be carried out.

\section{Experimental results}

The SAR imaging process [11] consists in analysing the signal collected by a moving radar and forming the spatial repartition of the illuminated ground. Whichever algorithm is used, the basic principle can be seen as a processing of the acquired data to obtain a focused bidimensional backscattering coefficient $H(k, \theta)$ for each illuminated point on the ground, and then to perform a Fourier-based spectral estimation to build the conventional SAR image $I(x, y)$ (see, for instance, the range migration algorithm (RMA) in [12,13]).
SAR imaging with wavelet transform allows construction of hyperimages in four dimensions $(x, y, f, \theta)$ which depend on two parameters $\left(\sigma_{f}, \sigma_{\theta}\right)$ of the mother wavelet $\phi(k, \theta)$. These two free parameters control the spread in frequency and in angular domain and play on inter-related resolutions in range, cross-range, frequency and angle.

The first analysed image represents an aircraft type target on the ground illuminated in X-band between $\left[-6^{\circ}, 6^{\circ}\right.$. The image constructed by the RMA algorithm is presented in Fig. $3 a$. On this image, the skeleton of the aircraft has been superimposed. The dimension of the image is about $30 \mathrm{~m}^{2}$ with a spatial resolution of $30 \mathrm{~cm}$. The second image, given in Fig. $3 b$ represents a building with some pylons around it in X-band for the same angular extent. The dimension of the area is $50 \mathrm{~m}^{2}$ with a spatial resolution of $30 \mathrm{~cm}$. The data have been collected by the RAMSES airborne SAR facilities [5].
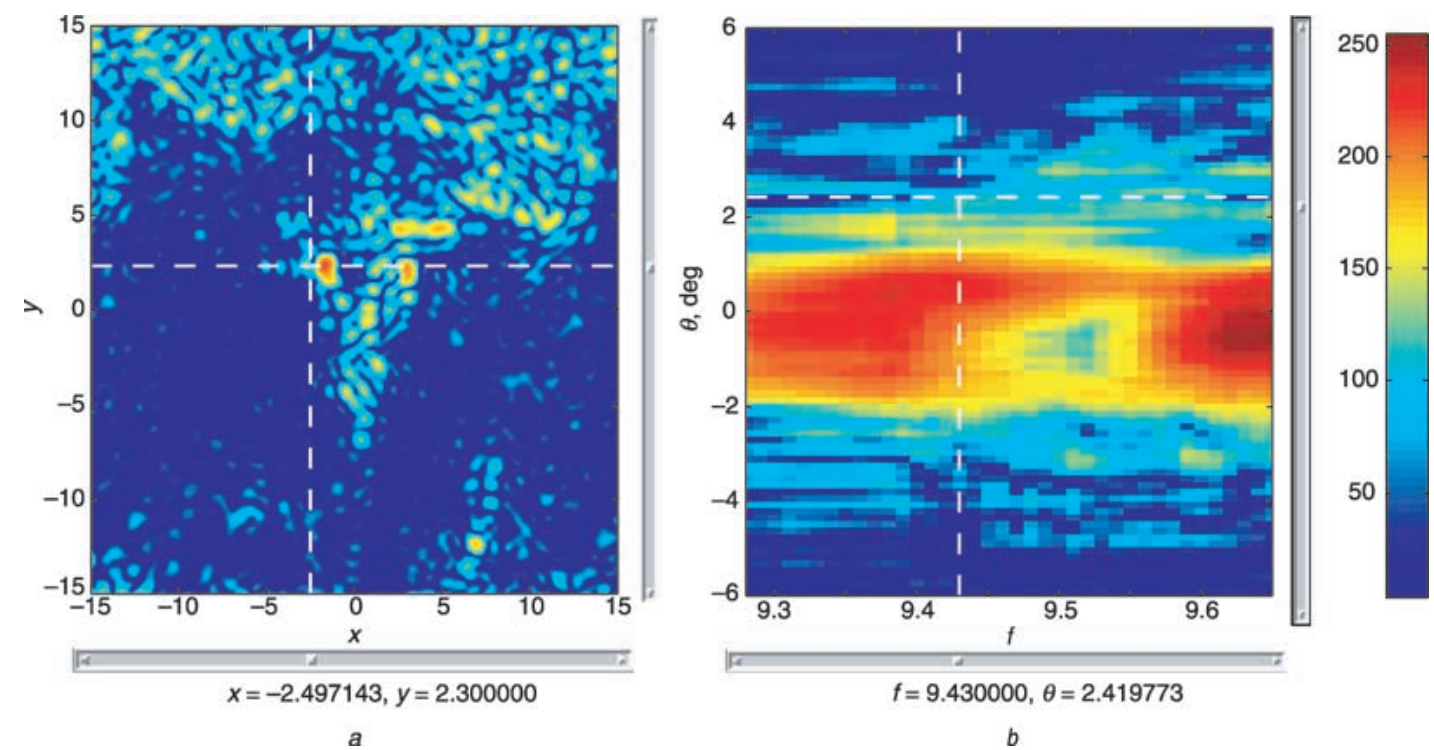

Fig. 6 Variation of spatial SAR image for a given angle $\theta=2.41^{\circ}$ and frequency $f=9.43 \mathrm{GHz}$

$a$ Image $(x, y)$

$b$ Image $(f, \theta)$ 

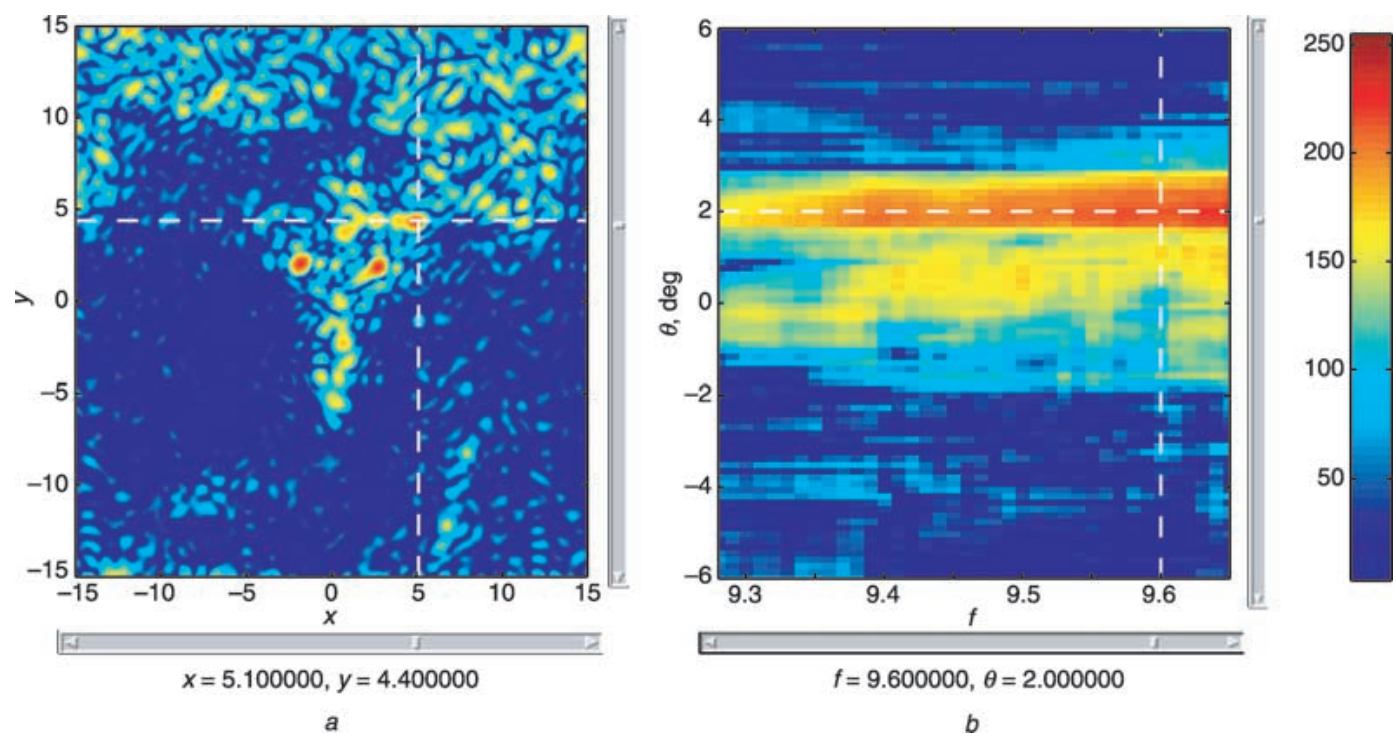

Fig. 7 Frequency and angular behaviour of a given reflector located at $(x=5.10 \mathrm{~m}, y=4.40 \mathrm{~m})$ : spatial image for $f=9.6 \mathrm{GHz}$ and $\theta=-2^{\circ}$

$a$ Image $(x, y)$

$b$ Image $(f, \theta)$

Figures 4, 5 and 6 show the sensitivity of the spatial SAR reflectors for three different angles of illumination $\left(\theta=-5.86^{\circ},-1.17^{\circ}\right.$ and $2.41^{\circ}$, respectively) for the same frequency $f=9.43 \mathrm{GHz}$. The analysis is made to obtain relative good resolution in range, cross-range, frequency and angle. Each Figure shows in the left-hand image the spatial repartition of the scatterers which are reflecting for the frequency and angle given by the cross in the right-hand image. Each of the right-hand images represents the distribution $(f, \theta)$ of the reflector located by the cross in the corresponding left-hand image.

Figure 4 shows the SAR spatial scatterers reflecting only for $\theta=-5.86^{\circ}$. For this angle of presentation, the characteristic form of the delta wing cannot be yet distinguished. The left trailing edge appears in Fig. 5 for an angle $\theta=-1.17^{\circ}$. The right edge appears in Fig. 6 for an angle $\theta=2.41^{\circ}$. This Figure characterises the property of nonisotropy of some reflectors in the whole angular domain $\left[-6^{\circ}, 6^{\circ}\right]$.

Figures 7 and 8 show the sensitivity in frequency and angular domain of different reflectors of the SAR image and the behaviour of the spatial image for two different points of the $(f, \theta)$ space.

Figure $9 a$ shows the SAR spatial scatterers reflecting only for $\theta=0^{\circ}$ and $f=9.6 \mathrm{GHz}$. In Fig. $9 b$, the frequency and angular behaviour of the bottom of a pylon is shown. It can be seen that this point is not isotropic.

Figure $10 a$ shows the SAR spatial scatterers reflecting only for $\theta=-4.8^{\circ}$ and $f=9.6 \mathrm{GHz}$. In Fig. $10 b$, the frequency and angular behaviour of the shadow of the building is shown. It can be seen that the shadow which represents only the thermal noise is white in frequency and is isotropic.
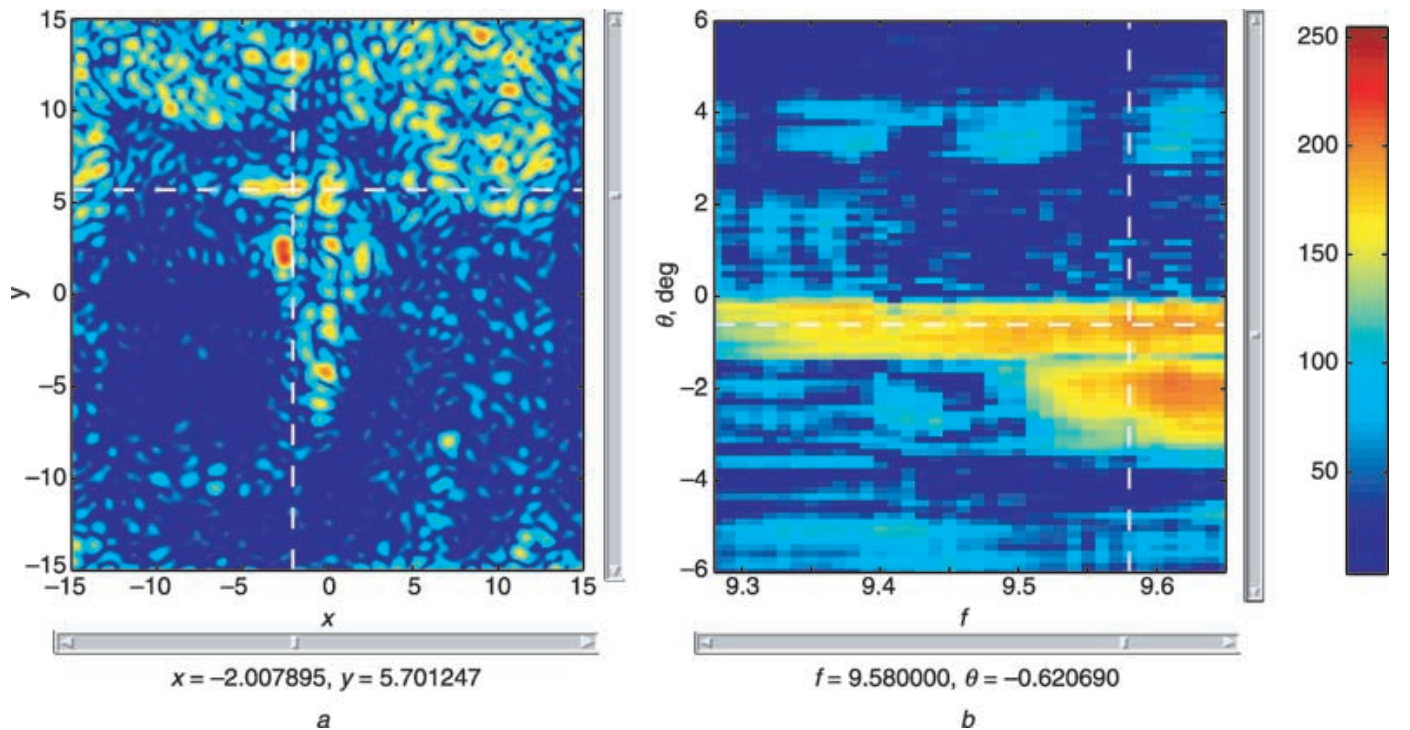

Fig. 8 Frequency and angular behaviour of a given reflector located at $(x=-2 \mathrm{~m}, y=5.70 \mathrm{~m})$ : spatial image for $f=9.58 \mathrm{GHz}$ and $\theta=-0.62^{\circ}$

$a$ Image $(x, y)$

$b$ Image $(f, \theta)$ 

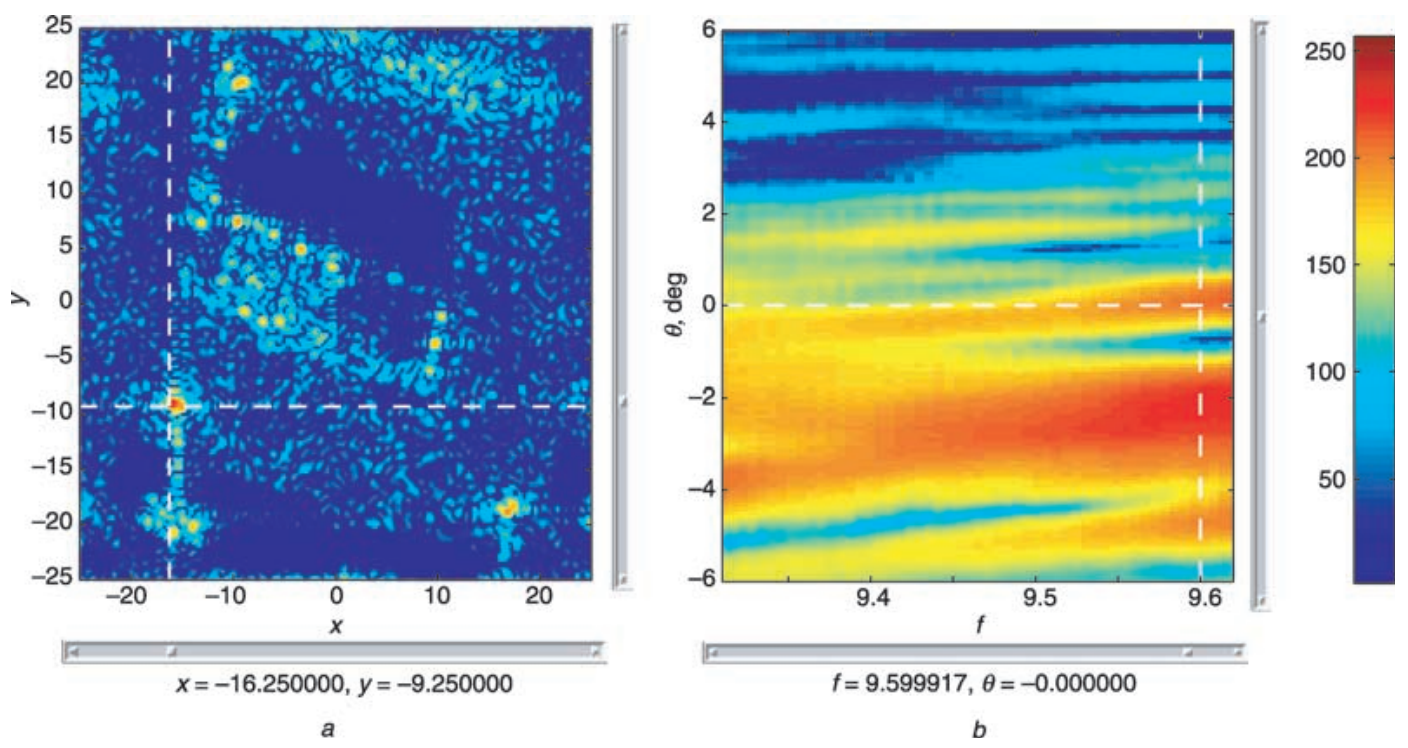

Fig. 9 Frequency and angular behaviour of a bottom of a pylon located at ( $x=-16.25 \mathrm{~m}, y=-9.25 \mathrm{~m}$ )

a Image $(x, y)$

$b$ Image $(f, \theta)$
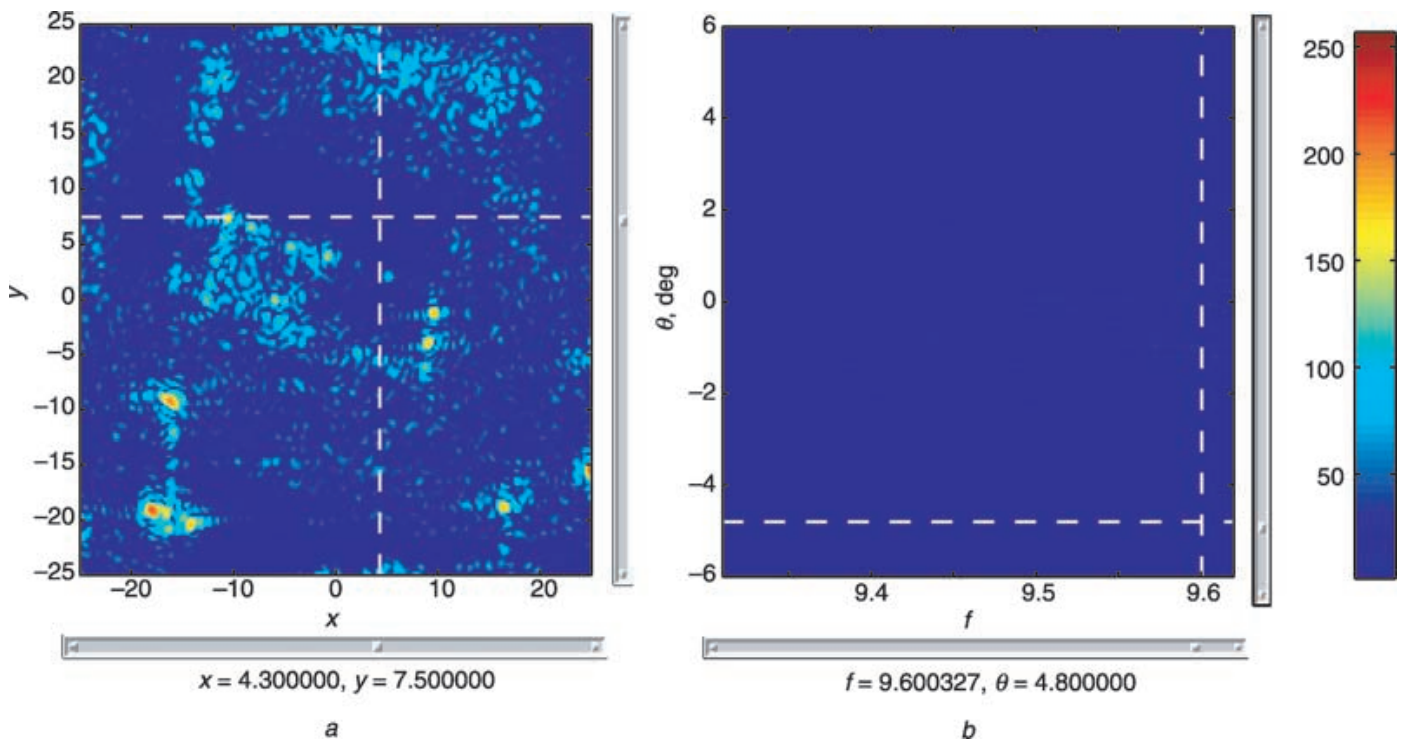

Fig. 10 Frequency and angular behaviour of the shadow of a building

a Image $(x, y)$

$b$ Image $(f, \theta)$

\section{Conclusions}

The theory of wavelet radar imaging was developed in [1] and applied to data collected in the laboratory (anechoic chamber) for different frequencies and angles of presentation, i.e. in spotlight configuration. This method has been extended to SAR data collected in another way (stripmap configuration). The multidimensional wavelet transform analysis for the SAR imaging can highlight some effects that classical SAR imaging cannot characterise. The first results of wavelet analysis on RAMSES SAR data showed that, even for a narrowband signal ( $6 \%$ of relative bandwidth) and for a middle angular extent of $12^{\circ}$, some reflectors are coloured and nonisotropic. These effects should be intensified with the emergence of high-resolution SAR image processes which require larger angular and spectral bandwidth. This is also the case for P-band SAR data used for foliage penetration.

This method of imaging allows more precise target recognition and identification, using its behaviour in the frequency and angular domains, than in the case of the multilook SAR processing, method, which only makes it possible to give poor angular sensitivity. Future work relates to the extraction of physical target attributes and to the use of wavelet transforms for imaging and detection of moving targets in SAR.

\section{References}

1 Bertrand, J., Bertrand, P., and Ovarlez, J.P.: 'Frequency directivity scanning in laboratory radar imaging', Int. J. Imaging Syst. Technol. $1994, \mathbf{5}$, pp. $39-51$ 
2 Vignaud, L.: 'Imagerie micro-ondes des scènes instationnaires'. Thèse de Doctorat de l'Université de Paris 6, France, 1996

3 Bertrand, J., and Bertrand, P.: 'Microwave imaging of time-varying target', Inverse Probl., 1997, 13, (3), pp. 621-645

4 Titin-Schnaider, C.: 'Discriminating polarimetric parameters for radar images analysis'. Proc. IGARSS'99, Hamburg, Germany, 28 June-2 July 1999

5 Boutry, J.M.: 'ONERA airborne SAR facilities'. Presented at 2nd Int. Airborne Remote Sensing Conf., San Francisco, CA, USA, June 1996

6 Mensa, D.L.: 'High resolution radar imaging' (Artech House, USA, 1981)

7 Vignaud, L., and Ovarlez, J.P.: 'Generalized wavelet for radar imaging', unpublished work
8 Bertrand, J., Bertrand, P., and Ovarlez, J.P.: 'Dimensionalized wavelet transform with application to radar imaging'. Proc. IEEE-ICASSP, Toronto, Canada, May 1991

9 Bertrand, J., and Bertrand, P.: 'The concept of hyperimage in wide-band radar imaging', IEEE Trans. Geosci. Remote Sens., 1996, 34 pp. 1144-1150

10 Castelli, J.C., and Bobillot, G.: 'i4D: a new approach to RCS imaging analysis'. Proc. AMTA, Boston, MA, USA, Nov. 1997

11 Carrara, W.G., Goodman, R.S., and Majewski, R.M.: 'Spotlight synthetic aperture radar' (Artech House, Boston, London)

12 Soumekh, M.: 'Fourier array imaging' (PTR Prentice-Hall, Englewood Cliffs, 1994)

13 Soumekh, M.: 'Synthetic aperture radar signal processing' (John Wiley \& Sons, Inc.) 